\title{
An Extra Cysteine in One of the Non-calcium-binding Epidermal Growth Factor-like Motifs of the FBN1 Polypeptide Is Connected to a Novel Variant of Marfan Syndrome
}

\author{
Christina Ståhl-Hallengren, * Tarja Ukkonen, ${ }^{*}$ Katariina Kainulainen, ${ }^{\star}$ Ulf Kristofersson, ${ }^{5}$ Tore Saxne, * Kristina Tornqvist, \\ and Leena Peltonen* \\ * Department of Rheumatology, Lund University Hospital, Sweden; ${ }^{\ddagger}$ Department of Human Molecular Genetics, National Public Health \\ Institute, 00300 Helsinki, Finland; and "Department of Clinical Genetics, and "Department of Opthalmology, Lund University Hospital, \\ 22185 Sweden
}

\begin{abstract}
We present here a family with a clinical phenotype resembling Marfan syndrome (MFS), and displaying joint contracture and episodes of knee joint effusions, but lacking the cardiovascular features of the syndrome. The phenotype of this family represents a unique mixture of connective tissue symptoms, some of which are found in classical MFS and some of which are typical of dominant ectopia lentis.

Linkage analyses suggested a linkage (LOD score 2.4; $\theta$ $=0$ ) between the phenotype of the family and a polymorphic marker in the vicinity of the fibrillin locus on chromosome 15 (FBN1). Furthermore, a novel transition mutation was identified in the FBN1 gene in all the affected members of the family. In contrast to the majority of fibrillin mutations reported so far, this mutation substitutes a cysteine for arginine, producing an extra cysteine in one of the non-calciumbinding EGF-like motifs of the fibrillin polypeptide, most probably disturbing the formation of one of the three disulfide bridges known to be essential for the normal conformation of this motif. (J. Clin. Invest. 1994. 94:709-713.) Key words: chromosome 15 - fibrillin • connective tissue • ectopia lentis • non-calcium-binding EGF-like domain
\end{abstract}

\section{Introduction}

Marfan syndrome (MFS) ${ }^{1}$ is a dominantly inherited connective tissue disorder with a prevalence of at least 4-6 cases per 100,000 population (1). It is caused by mutations in the gene coding for an essential component of connective tissue microfibrils, fibrillin 15 (FBN1) (2-5). To date, over 30 different mutations have been reported in numerous Marfan patients,

Address all correspondence to to Leena Peltonen, M.D., Ph.D., National Public Health Institute, Department of Human Molecular Genetics, Mannerheimintie 166, FIN-0300 Helsinki, Finland.

Received for publication 22 December 1993 and in revised form 19 April 1994.

1. Abbreviations used in this paper: EL, ectopia lentis; FBN1, fibrillin gene on chromosome 15; FBN2, fibrillin gene on chromosome 5; MFS, Marfan syndrome; non-cb EGF-like domain, non-calcium-binding EGF-like domain.

J. Clin. Invest.

(c) The American Society for Clinical Investigation, Inc.

0021-9738/94/08/0709/05 $\$ 2.00$

Volume 94, August 1994, 709-713 the majority of them disturbing the calcium-binding EGF-like domains of this polypeptide $(2,6-13)$.

The clinical characteristics of the classical form of MFS include lens dislocation, aortic root dilatation and dissection, cusp insufficiency, and skeletal manifestations such as arachnodactyly dolichostenomelia, thoracic deformities, and increased joint laxity (1). However, the symptoms are not always pronounced and diagnosis may be difficult in mild cases. Furthermore, there is considerable variation in the clinical phenotype between families and also within the same family. When suspected, a careful hereditary study and thorough physical examination of the patient and his/her relatives is often conclusive, but diagnosis may be difficult in cases with few atypical symptoms and also in sporadic cases (1).

Two other related clinical entities have also been suggested as being caused by defects in the fibrillin gene on chromosome 15 (FBN1) or in a highly homologous gene on chromosome 5. Families demonstrating dominant ectopia lentis (EL) with eye symptoms and some skeletal features of MFS, but no cardiovascular symptoms, have been genetically linked to FBN1 and the first mutation has recently been identified (14-16). Another related clinical phenotype, congenital contractural arachnodactyly, has been linked to the fibrillin gene on chromosome 5 (FBN2) but so far no specific mutation has been identified (17).

\section{Methods}

The proband. A 21-yr-old male with sphaerofacia and lens dislocation since childhood was referred to the Department of Rheumatology with a preliminary diagnosis of MFS. He suffered from hand pain, especially during motion, and from episodes of knee joint effusions, but the range of motion was normal and no joint deformities or other signs of arthritis were present. He had no scoliosis and denied cardiac symptoms. Since several relatives had similar signs and symptoms, we decided to perform a clinical and genetic study of the family to elucidate whether the condition represented a variant of MFS or a separate syndrome characterized by lens dislocation and joint symptoms.

14 out of 19 family members in three generations were examined by the same investigator. Of the persons not examined, three were living abroad, one was being treated elsewhere for a lymphoma and one was not willing to participate. The 14 participants were examined for joint deformities, range of motion, and synovitis. The presence of scoliosis and other thoracic deformities was registered and a physical examination of the heart was made. Seven of the eight family members with lens dislocation underwent routine echocardiography (cardiovascular ultra sound) with particular attention to aortic root dimensions and valvular function. Family member III/ 12 refused both this examination and blood sampling. Characteristics of MFS were registered; these included a high arched palate, arachnodactyly, an increased arm span and abnormal proportions between the upper and lower body segments (1). Medical 


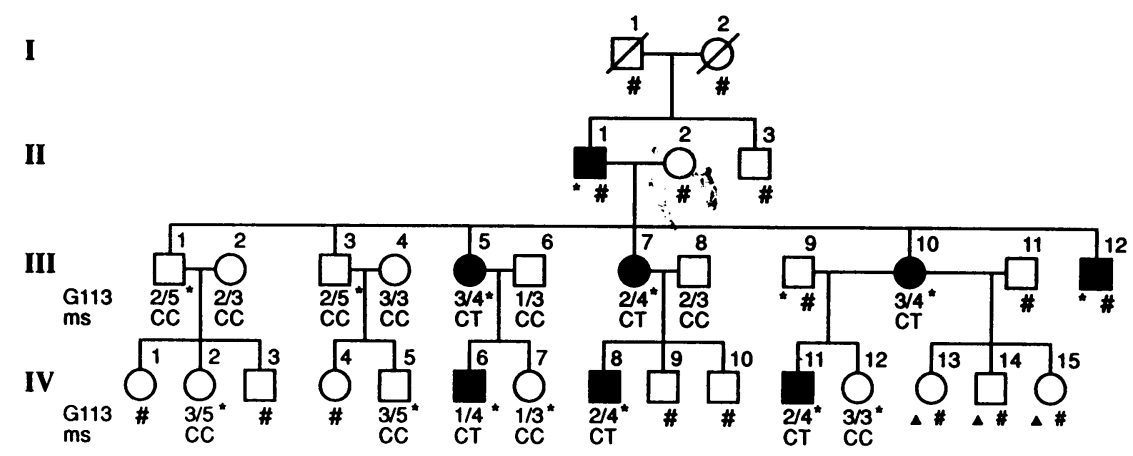

Figure 1. Pedigree of the family. The genotypes of each individual with the G113 marker and minisequencing results $(\mathrm{ms})$ detecting the R122C mutation are shown below each individual. Each affected family member carries a $\mathrm{C}_{364}$ to-T transition. $(*)$ Clinically examined individuals; ( $\Delta$ ) subjects below six years of age; (\#) subjects whose samples were not available. records from the Department of Opthalmology and primary care physicians were examined for mentions of lens dislocation and joint and cardiac symptoms.

DNA analyses. Genomic DNA was extracted by standard procedures from the peripheral blood leucocytes of 16 individuals in two generations (18). In the case of one affected family member, genomic DNA was extracted from cultured fibroblasts. DNA samples were amplified using primers specific for polymorphisms of FBN1 (17), G113 (19), and CYP 19 (20), which are located in or close to the FBN1 locus on chromosome 15. PCR amplification and subsequent analyses of the amplified products were performed as described previously (21). Linkage analyses were performed using the computer programs MLINK and LINKMAP of Linkage Package 5.0 (22).

A fibrillin-specific primer covering nucleotides 5'-1992-1901 (the nucleotides are numbered in accordance with the amino acid sequence reported by Pereira et al.) (23) was used to synthesize FBN1 cDNA by reverse transcriptase from the total RNA extracted from the cultured fibroblasts (24). $2 \mu \mathrm{l}$ of the resulting cDNA were PCR amplified (25) using a primer corresponding to nucleotides 5'-710-691 (biotinylated at its $5^{\prime}$-end) and another primer covering nucleotides 5'-231-254. Sequencing of the amplification product was performed as previously described with modifications made for biotinylated primers and the automated DNA sequencing system (Pharmacia, Uppsala, Sweden) (26, 27). A 5' fluorescein-labeled primer covering nucleotides 5'-231-254 of FBN1 cDNA was used as a sequencing primer (28).

The mutation was screened for in the genomic DNA of 16 family members using the solid-phase minisequencing technique $(29,30)$. For solid-phase minisequencing genomic DNA was amplified using a 5' biotinylated primer covering nucleotides $5^{\prime}-441-420$ and a primer covering nucleotides $5^{\prime}-347-363$ and seven nucleotides from the previous intron (tttcaagTACAACACTGCAATATT). The amplified 5' biotinylated PCR product was immobilized on an avidin matrix and rendered single stranded. The mutated nucleotide in the immobilized DNA was identified by a one-step primer extension reaction, directed by a detection step primer covering nucleotides $5^{\prime}-347-363$ and seven nucleotides from the previous intron, which anneals to the DNA immediately upstream of the site of variation $(29,30)$.

The consequences of the extra cysteine for the second non-calciumbinding EGF-like motif of the fibrillin polypeptide were predicted using the Betaturn (31) and Novotny (32) computer programs of the Intelligenetic PC gene software package.

\section{Results}

Fig. 1 shows a pedigree of four generations of the family. The pedigree is compatible with autosomal dominant inheritance. All the eight individuals with lens dislocation also had joint symptoms, while the rest had neither of the symptoms. Table I shows clinical data for those eight individuals. No signs of aortic root dilatation, mitral valve prolapse, or any kind of cardiac involvement were observed neither on physical examination nor on echocardiogram (cardiovascular ultrasound) in any of the family members and there was no history of sudden deaths in the family. A 63-yr-old man who had mild angina pectoris was the only family member with any cardiac symptoms. One or several episodes of knee joint effusion with moderate pain had occurred in five individuals. These episodes may have been related to moderate physical activity. The physical examination in this study did not reveal ongoing joint effusion or other signs of synovitis in any of the family members. Five individuals had flexion contractures of the fifth proximal interphalangeal joint, whereas none of the healthy family members had this. Four adults and one child had extension defects in their elbows. Otherwise joint mobility was normal and increased joint laxity was not noted. Wrist and thumb tests were normal. The median ratio of the upper and lower body segments was 0.86 (range 0.83-0.89), which is within the range characteristic of MFS.

We analyzed whether there was cosegregation of the clinical phenotype and three polymorphic markers located inside or in

Table I. Clinical Characteristics of the Affected Family Members

\begin{tabular}{lll}
\hline & $\delta(5)$ & \\
\hline Skeletal involvement & & \\
Scoliosis & 1 & 2 \\
Thoracal kyphosis & 1 & 2 \\
Pectus exacavatum & 1 & 1 \\
High arched palate & 1 & 1 \\
"Thumb sign" & 0 & 0 \\
"Wrist sign" & 0 & 0 \\
Arm span $\geq 8$ cm & 1 & 2 \\
Upper segment/lower segment & Median & 0.86 (range $0.83-0.89)$ \\
Joint symptoms & & \\
Joint laxity & 0 & 0 \\
Morning stiffness of joints $>1$ h & 0 & 1 \\
Extension defects, dig "V" & 2 & 3 \\
Extension defects, elbow & 3 & 2 \\
Pains in hands & 2 & 3 \\
Knee joint effusion & 3 & 2 \\
Arthrocentesis performed & 1 & 2 \\
Meniscectomy performed & 1 & 1 \\
Eye symptoms & & 3 \\
Lens dislocation & 5 & 0 \\
Other organs & & \\
Signs of cardiac involvement & 0 & (bilat) \\
Inguinal hernia & & \\
& &
\end{tabular}



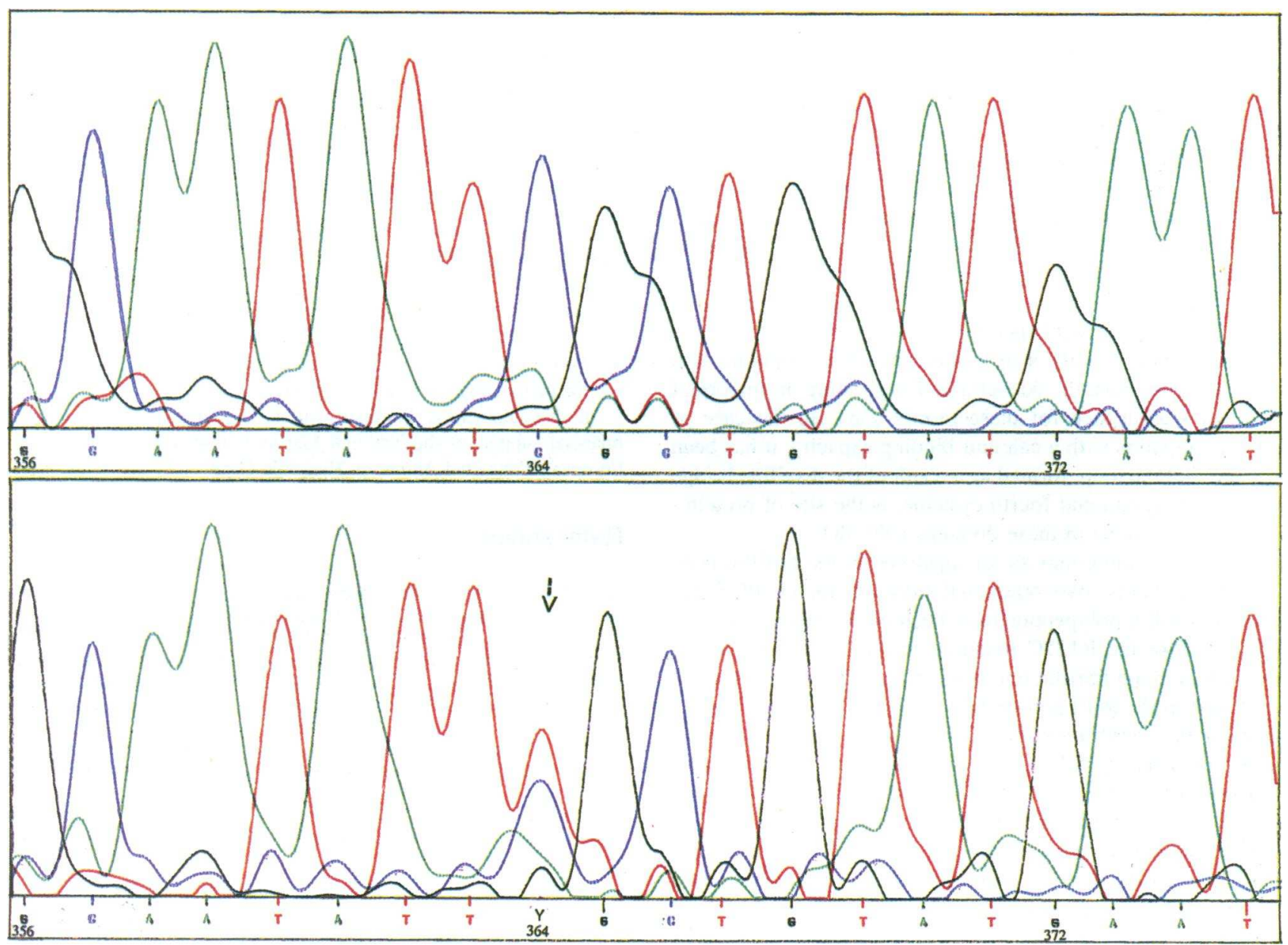

Figure 2. Detection of the $\mathrm{C}_{364}$-to-T point mutation in fibrillin cDNA using the automated DNA sequencer. Arrow indicates the heterozygote in position 364 .

the vicinity of the fibrillin locus on chromosome 15 in this family. Two of the markers, FBN1 and CYP19, were noninformative, whereas linkage analysis of the third marker, G113, revealed a LOD score of 2.4 with no recombinations between the disorder and the marker. This finding strongly suggested that the clinical phenotype seen in this family was caused by a defect in the FBN1 gene.

The mRNA extracted from the cultured fibroblasts of family member III/7 was included in our search for mutations in the coding sequence of the FBN1 gene. By using the automated sequenator in the screening of specific regions of FBN1 cDNA, a C to $\mathrm{T}$ transition was detected at nucleotide 364 in the FBN1 cDNA of this patient. This mutation substitutes codon 122 coding for cysteine for one coding for arginine (R122C) (Fig. 2).

The mutation was confirmed in the genomic DNA of the proband using the solid-phase minisequencing technique, which unequivocally identifies the mutated nucleotide $(29,30)$. Using the same technique, all the affected individuals in the family were shown to carry the mutation, whereas it was not detected in the DNA of the unaffected members of the family (Fig. 1). Furthermore, none of the 60 Marfan patients or 60 healthy controls analyzed were shown to carry this mutation.

The fibrillin polypeptide chain is made up of 47 repetitive EGF-like repeats interspersed by other motifs. It has been stated that the majority of EGF-like motifs have calcium-binding properties, whereas three of them located close to the aminoterminal end of the fibrillin polypeptide exhibit the characteristic sixcysteine pattern, but lack the putative calcium-binding consensus sequence $(33,34)$. The $\mathrm{R} 122 \mathrm{C}$ mutation of this family occurs in the second of these three non-calcium-binding EGFlike (non-cb EGF-like) motifs and produces an extra cysteine just prior to the conserved second cysteine in this motif. The second cysteine normally participates in one of the three disulfide bridges essential for antiparallel $\beta$-sheet conformation of the EGF-like motif. Except for providing an extra potentially reactive cysteine, the computer-assisted predictions for the consequences of this mutation suggested a drop in local hydrophobicity and a change in the isoelectric point of this particular EGF-like motif from 7.01 to 6.26 due to the replacement of a neutral cysteine with a basic arginine.

\section{Discussion}

We report here a novel FBN1 mutation which is located in one of the non-cb EGF-like motifs of fibrillin polypeptide and causes the substitution of an extra cysteine for arginine just before the second cysteine of this six-cysteine motif. Due to its close vicinity to a cysteine participating in disulfide bridge 
formation, this extra cysteine most probably results in some "wobbling" in the formation of the disulfide bridge between the second and fourth cysteine and consequently disturbs the proper $\beta$-sheet conformation of this particular EGF-like motif. Although precise prediction of the consequences of an additional cysteine in this motif is difficult, any interference in normal disulfide bridge formation will most probably change the structure of the motif and have long-range structural effects on the native protein, especially since the disulfide-dependent aggregation of FBN1 is necessary for normal microfibrillar assembly (35).

EGF-like motifs with invariant six-cysteine residues have been identified in a wide array of human, other vertebrate, and invertebrate proteins, the conservation suggesting an important functional significance for these motifs (36). In the case of EGF-like domains with a calcium-binding capacity, it has been proposed that the loop, formed as a result of the disulfide bridge between the second and fourth cysteine, is the site of proteinreceptor interactions in these domains $(37,38)$.

Of some 30 mutations so far identified in the fibrillin gene in Marfan patients, two other mutations in non-cb EGF-like motifs of fibrillin polypeptide have been mentioned in congress reports. Unlike the $\mathrm{R} 122 \mathrm{C}$ mutation located in the second of these motifs in the fibrillin polypeptide, both of these mutations are located in the third non-cb EGF-like motif. One substitutes serine for the fourth cysteine (C166S) (39) and the other replaces this same cysteine with phenylalanine (C166F) (40). As opposed to the R122C mutation, both of these mutations result in the absence of the disulfide bond between the second and fourth cysteine. The patients carrying the C166F or C166S mutation in the third non-cb EGF-like motif were reported to represent the classical phenotype of MFS, whereas the family described here represents a clinical phenotype which resembles MFS, but totally lacks the cardiovascular features considered essential for a clinical diagnosis of MFS. Furthermore, in contrast to the commonly observed joint hypermobility, the patients with the R122C mutation developed joint contractures and suffered from episodes of knee joint effusions somewhat resembling two previously described families in which EL was associated with dolichostenomelia and joint stiffness (41). It is worth noting that the symptoms and signs have remained more or less unchanged over the three generations studied, which contrasts with the more variable phenotypic expression of MFS normally observed (1). Altogether, these mutations, occurring in EGFlike motifs with no calcium-binding properties, demonstrate that disturbance in the calcium-binding capacity of EGF motifs is not the only reason for a marfanoid phenotype.

The clinical phenotype in the family described here bears some similarities to the phenotype of dominant EL. The first mutation of FBN1 (E2447K), resulting in the EL phenotype with eye and skeletal involvement but no cardiovascular findings, has recently been identified (16). Unlike the mutation described here, the E2447K mutation affects the carboxyterminal end of the fibrillin polypeptide, and substitutes a highly conserved glutamic acid residue in one of the calcium-binding EGF-like motifs. As in the case of all the 30-plus FBN1 mutations characterized so far in MFS patients, no rules can yet be given regarding the genotype and the resulting phenotype (2, $6-13)$. The two previously described mutations in the non-cb EGF-like motifs both remove an essential disulfide bridge in the motif and result in the classical Marfan phenotype, whereas the mutation presented here and the only EL mutation reported to date do not directly remove the disulfide bridge. Whether this difference goes some way to explaining why the latter cases do not display any symptoms in cardiovascular tissues, which face the highest mechanical stress and most demanding requirements for tissue elasticity, remains to be clarified with further identification of mutations in individuals with Marfanoid phenotypes but very mild or nonexistent cardiovascular symptoms.

\section{Acknowledgments}

We wish to thank Dr Rantamäki for the analyses of the DNA samples. We thank Lotta Larsson for assisting in collecting the blood samples and Dr Jan Eskilsson, Department of cardiology, Lund University Hospital, for performing the echocardiograms.

K. Kainulainen, L. Peltonen, and T. Ukkonen are grateful for the financial support of the National Marfan Foundation of the USA and European Concerted Action on Heritable Connective Tissue Disorders.

\section{References}

1. Pyeritz, R. E. 1993. The Marfan Syndrome. In Connective Tissue and Its Heritable Disorders. Royce, P. M., B. Steinmann, editors. Wiley-Liss. 437-468. 2. Dietz, H. C., G. R. Cutting, R. E. Pyeritz, C. L. Maslen, L. Y. Sakai, G. M. Corson, E. G. Puffenberger, A. Hamosh, E. J. Nanthakumar, S. M. Curristin, G. Stetten, D. A. Meyers, and C. A. Francomano. 1991. Marfan syndrome caused by a recurrent de novo missense mutation in the fibrillin gene. Nature (Lond.) 352:337-339.

3. Kainulainen, K., L. Pulkkinen, A. Savolainen, I. Kaitila, and L. Peltonen. 1990. Location on chromosome 15 of the gene defect causing Marfan syndrome. N. Engl. J. Med. 323:935-939.

4. Magenis, R. E., C. L. Maslen, L. Smith, L. Allen, and L. Y. Sakai. 1991. Localization of the fibrillin (FBN) gene to chromosome 15, band q21.1. Genomics. 11:346-351.

5. Sakai, L. Y., D. R. Keene, and E. Engvall. 1986. Fibrillin, a new 350-kD glycoprotein, is a component of extracellular microfibrils. J. Cell Biol. 103:24992509.

6. Dietz, H. C., R. E. Pyeritz, E. G. Puffenberger, R. J. Kendzior Jr, G. M. Corson, C. L. Maslen, L. Y. Sakai, C. A. Francomano, and G. R. Cutting. 1992. Marfan phenotype variability in a family segregating a missense mutation in the epidermal growth factor-like motif of the fibrillin gene. J. Clin. Invest. 89:16741680.

7. Dietz, H. C., J. M. Saraiva, R. E. Pyeritz, G. R. Cutting, and C. A. Francomano. 1992. Clustering of fibrillin (FBN1) missense mutations in Marfan syndrome patients at cysteine residues in EGF-like domains. Human Mutation. 1:366374.

8. Kainulainen, K., L. Y. Sakai, A. Child, F. M. Pope, L. Puhakka, L. Ryhänen, A. Palotie, I. Kaitila, and L. Peltonen. 1992. Two mutations in Marfan syndrome resulting in truncated fibrillin polypeptides. Proc. Natl. Acad. Sci. USA. 89:59175921.

9. Dietz, H. C., D. Valle, C. A. Francomano, R. J. Kendzior Jr., R. E. Pyeritz and G. R. Cutting. 1993. The skipping of constitutive exons in vivo induced by nonsense mutations. Science (Wash. DC). 259:680-683.

10. Dietz, H. C., I. McIntosh, L. Y. Sakai, G. M. Corson, S. C. Chalberg R. E. Pyeritz, and C. A. Francomano. 1993. Four novel FBN1 mutations: significance for mutant transcript level and EGF-like domain calcium binding in the pathogenesis of Marfan syndrome. Genomics. 17:468-475.

11. Godfrey, M., N. Vandemark, M. Wang, M. Velinov, D. Wargowski, P. Tsipouras, J. Han, J. Becker, W. Robertson, S. Droste, and V. H. Rao. 1993. Prenatal diagnosis and a donor splice site mutation in fibrillin in a family with Marfan syndrome. Am. J. Hum. Genet. 53:472-480.

12. Hewett, D. R., J. R. Lynch, R. Smith, and B. C. Sykes. 1993. A novel fibrillin mutation in the Marfan syndrome which could disrupt calcium binding of the epidermal growth factor-like module. Hum. Mol. Genet. 2:475-477.

13. Kainulainen, K., L. Karttunen, L. Puhakka, L. Sakai, and L. Peltonen. 1994. Mutations in the fibrillin gene responsible for dominant ectopia lentis and neonatal Marfan syndrome. Nature Genetics. 6:64-69.

14. McKusick, V. A. 1992. Mendelian Inheritance in Man. 10th edition. The Johns Hopkins University Press, Baltimore, MD. 334 pp.

15. Tsipouras, P., R. Del Mastro, M. Sarfarazi, B. Lee, E. Vitale, A. H. Child, M. Godfrey, R. B. Devereux, D. Hewett, B. Steinmann, D. Viljoen, B. C. Sykes, M. Kilpatrik, and F. Ramirez. 1992. Genetic linkage of Marfan syndrome, ectopia lentis and congenital contractural arachnodactyly to the fibrillin genes on chromosome 15 and 5. N. Engl. J. Med. 326:905-909.

16. Lönnqvist, L., A. Child, K. Kainulainen, R. Davidson, L. Puhakka, and 
L. Peltonen. 1994. A novel mutation of the fibrillin gene causing ectopia lentis. Genomics. 19:573-576.

17. Lee, B., M. Godfrey, E. Vitale, H. Hori, M. G. Mattei, M. Sarfarazi, P. Tsipouras, F. Ramirez, and D. W. Hollister. 1991. Linkage of Marfan syndrome and a phenotypically related disorder to two different fibrillin genes. Nature (Lond.). 352:330-334.

18. Vandenplas, S., I. Wiid, A. Grobler-Rabie, K. Brebner, M. Ricketts, G. Wallis, A. Bester, C. Boyd, and C. Mathew. 1984. Blot hybridization analysis of genomic DNA. J. Med. Genet. 21:164-172.

19. Hudson, T. J., M. Engelstein, M. K. Lee, E. C. Ho, M. J. Rubenfield, C. P. Adams, D. E. Housman, and N. C. Dracopoli. 1992. Isolation and chromosomal assignment of 100 highly informative human simple sequence repeat polymorphisms. Genomics. 13:622-629.

20. Polymeropoulos, M. H., H. Xiao, D. S. Rath, and C. R. Merril. 1991. Tetranucleotide repeat polymorphism at the human aromatase cytochrome P-450 gene (CYP19). Nucleic Acids Res. 19:195.

21. Rantamäki, T., L. Lönnqvist, L. Karttunen, and L. Peltonen. 1994. DNA diagnostics of the Marfan syndrome: application of amplifiable polymorphic markers. Eur. J. Hum. Gen. 2:66-75.

22. Lathrop, G. M., J. M. Lalouel, C. Julier, and J. Ott. 1984. Strategies for multilocus linkage analysis in humans. Proc. Natl. Acad. Sci. USA. 81:34433446.

23. Pereira, L., M. D'Alessimo, F. Ramirez, J. R. Lynch, B. Sykes, T. Pangilinan, and J. Bonadio. 1993. Genomic organization of the sequence coding for fibrillin, the defective gene product in Marfan syndrome. Hum. Mol. Genet. 2:961 968.

24. Chirgwin, J. M., A. E. Przybyla, R. J. MacDonald, and W. J. Rutter. 1979. Isolation of biologically active ribonucleic acid from sources enriched in ribonuclease. Biochemistry. 18:5294-5299.

25. Mullis, K. B., and F. A. Faloona. 1987. Specific synthesis of DNA in vitro via a polymerase-catalyzed chain reaction. Methods Enzymol. 155:335-350.

26. Sanger, F., S. Niclen, and A. R. Coulson. 1977. DNA sequencing with chain-terminating inhibitors. Proc. Natl. Acad. Sci. USA. 74:5463-5467.

27. Syvänen, A. C., K. Aalto-Setälä, K. Kontula, and H. Söderlund. 1989. Direct sequencing of affinity-captured amplified human DNA: application to the detection of apolipoprotein E polymorphism. FEBS (Fed. Eur. Biochem. Soc.) Lett. 258:71-74.

28. Ansorge, W., B. Sproat, J. Stegemann, C. Schwager, and M. Zenke. 1987. Automated DNA sequencing: ultrasensitive detection of fluorescent bands during electrophoresis. Nucleic Acids Res. 15:4593-4602.
29. Syvänen, A. C., K. Aalto-Setälä, L. Harju, K. Kontula, and H. Söderlund 1990. A primer guided nucleotide incorporation assay in the genotyping of apolipoprotein E. Genomics. 8:684-692.

30. Syvänen, A. C., E. Ikonen, T. Manninen, M. Bengström, H. Söderlund, P. Aula, and L. Peltonen. 1992. Convenient and quantitative determination of the frequency of a mutant allele using solid-phase minisequencing: Application to aspartylglucosaminuria in Finland. Genomics. 12:590-595.

31. Chou, P. Y., and G. D. Fasman. 1979. Prediction of $\beta$-turns. Biophys. J. 26:367-384.

32. Novotny, J., and C. Auffray. 1984. A program for prediction of protein secondary structure from nucleotide sequence data: application to histocompatibility antigens. Nucleic Acids Res. 12:243-255.

33. Maslen, C. L., G. M. Corson, B. K. Maddox, R. W. Glanville, and L. Y. Sakai. 1991. Partial sequence of a candidate gene for the Marfan syndrome. Nature (Lond.). 352:334-337.

34. Corson, G. M., S. C. Chalberg, H. C. Dietz, N. L. Charbonneau, and L. Y. Sakai. 1993. Fibrillin binds calcium and is coded by cDNAs that reveal a multidomain structure and alternatively spliced exons at the $5^{\prime}$ end. Genomics. 17:476-484.

35. Sakai, L. Y., D. R. Keene, R. W. Glanville, and H. P. Bächinger. 1991. Purification and partial characterization of fibrillin, a cysteine-rich structural component of connective tissue microfibrils. J. Biol. Chem. 266:14763-14770.

36. Wharton, K. A., K. M. Johansen, T. Xu, and S. Artavanis-Tsakonas. 1985 Nucleotide sequence from the neurogenic locus notch implies a gene product that shares homology with proteins containing EGF-like repeats. Cell. 43:567-581.

37. Appella, E., E. A. Robinson, S. J. Ullrich, M. P. Stoppeli, A. Corti, G. Cassani, and F. Blasi. 1987. The receptor-binding sequence of urokinase. J. Biol. Chem. 262:4437-4440.

38. Komoriya, A., M. Hortsch, C. Meyers, M. Smith, H. Kanety, and J. Schlessinger. 1984. Biologically active synthetic fragments of epidermal growth factor: localization of a major receptor-binding region. Proc. Natl. Acad. Sci. USA. 81:1351-1355.

39. Tilstra, D., and P. H. Byers. 1993. A novel mutation in a non-calciumbinding EGF-like motif in a family with the Marfan syndrome. Scientific workshop of the Marfan syndrome. Portland, Oregon. Abstract.

40. Dietz, H. C. 1994. Mutation Report in Marfan Syndrome and Related Disorders Research Consortium Database.

41. Stevenson, R. E., R. J. Schroer, H. A. Taylor, J. D. Compton, and R. E. Livingstone. 1982. Dislocated lens, dolichostenomelia, and joint stiffness. Proc. Greenwood Genet. Center. 1:16-22. 